Supporting Information for:

\title{
Computational Investigation of the Base Flipping of the Thymine Dimer in Duplex DNA
}

\author{
Lauren L O’Neil ${ }^{\dagger}$, Alan Grossfield ${ }^{\ddagger}$ and Olaf Wiest ${ }^{\dagger} *$ \\ ${ }^{\dagger}$ Department of Chemistry and Biochemistry, University of Notre Dame, \\ Notre Dame, Indiana 46556-5670 (USA) \\ ${ }^{\ddagger}$ I.B.M. T.J. Watson Research Center, Yorktown Heights, New York 10598 \\ owiest@nd.edu
}

RMSd vs. time for flipped-in and flipped-out conformations of DNA

RDF for water distribution around A24 and A25

$\mathrm{S} 2, \mathrm{~S} 3$

3 ns snaphot for water distribution around CPD

Average Occupancies and Angels for Water-CPD Interactions

Average Global Bends for Flipped-In and Flipped-Out Structures

Structure Snapshots at 3, 3.5 and 7.9 ns for Flipped-Out Structure

Sampling Scheme for PMF

Convergence plots fro PMF

S9-S11 


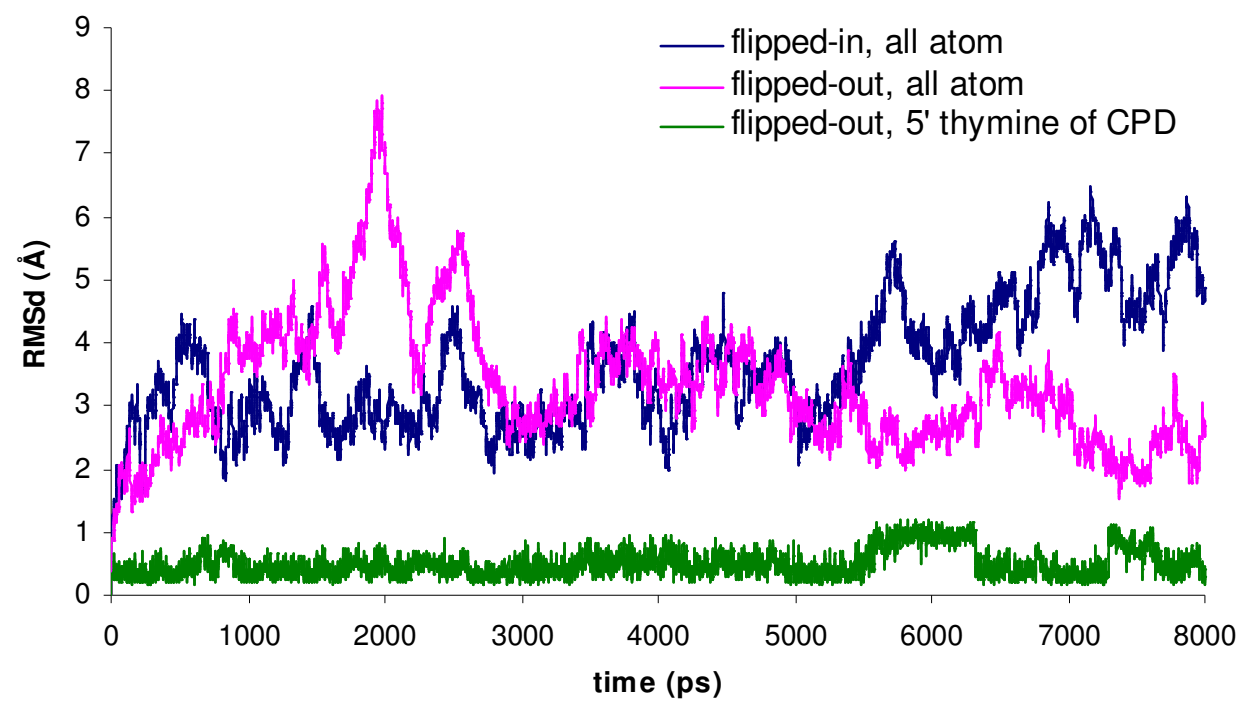

Figure S1: RMSd vs. time for flipped-in and flipped-out conformations of DNA. RMSd calculated from minimized, equilibrated structure over $8 \mathrm{~ns}$ of trajectory (data saved every $1 \mathrm{ps}$ ). "All atom" RMSd include all heavy atoms (C, N, O, P) but not hydrogens. RMSd from 5' thymine of CPD includes hydrogens.

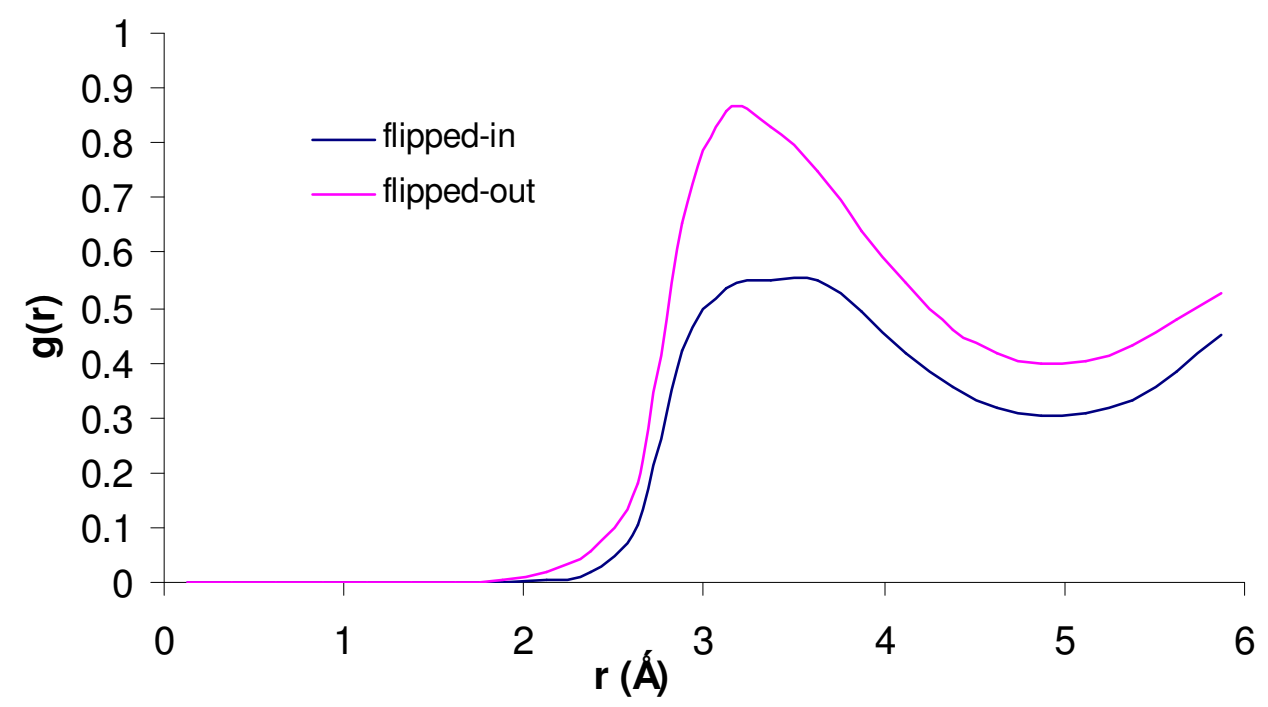

Figure S2. Thymine dimer containing DNA, flipped-in (blue) and flipped-out (pink), radial distribution function calculated over $8 \mathrm{~ns}$ MD production runs from A24 (opposite 5' thymine of thymine dimer) $\mathrm{N} 6\left(-\mathrm{NH}_{2}\right)$ to water. 


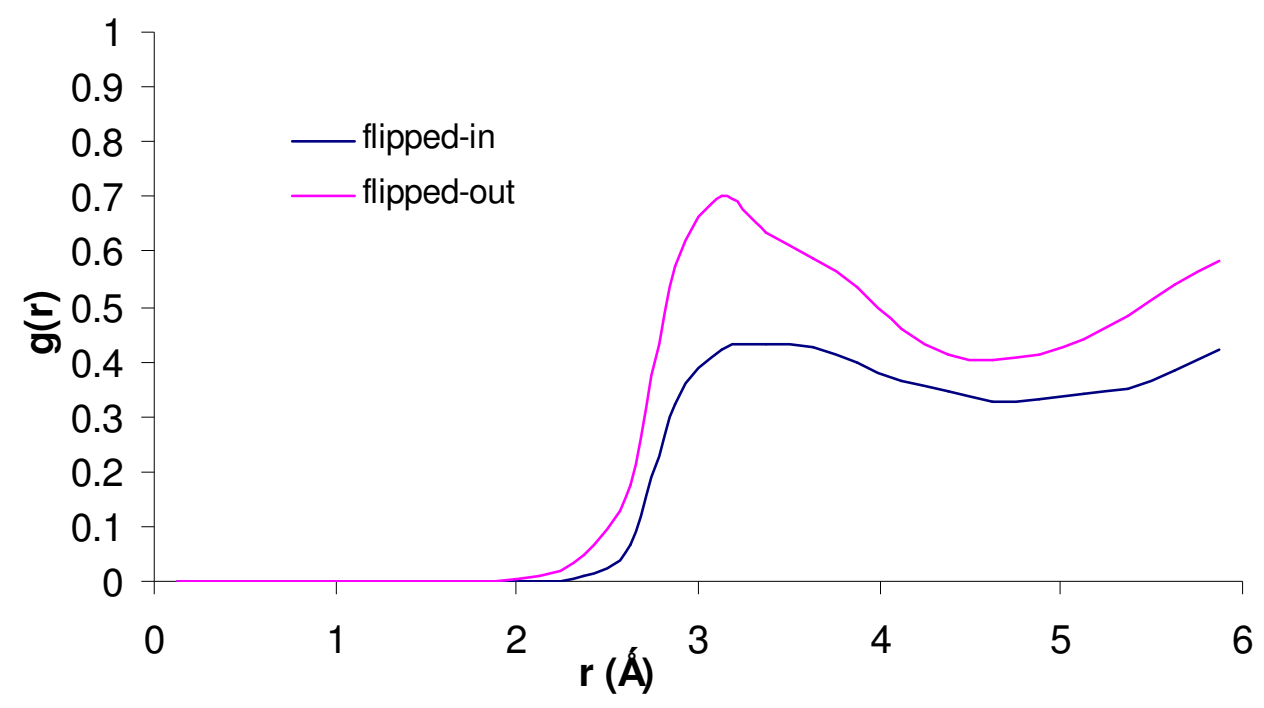

Figure S3. Thymine dimer containing DNA, flipped-in (blue) and flipped-out (pink), radial distribution function calculated over $8 \mathrm{~ns}$ MD production runs from A25 (opposite 5' thymine of thymine dimer) $\mathrm{N} 6\left(-\mathrm{NH}_{2}\right)$ to water.
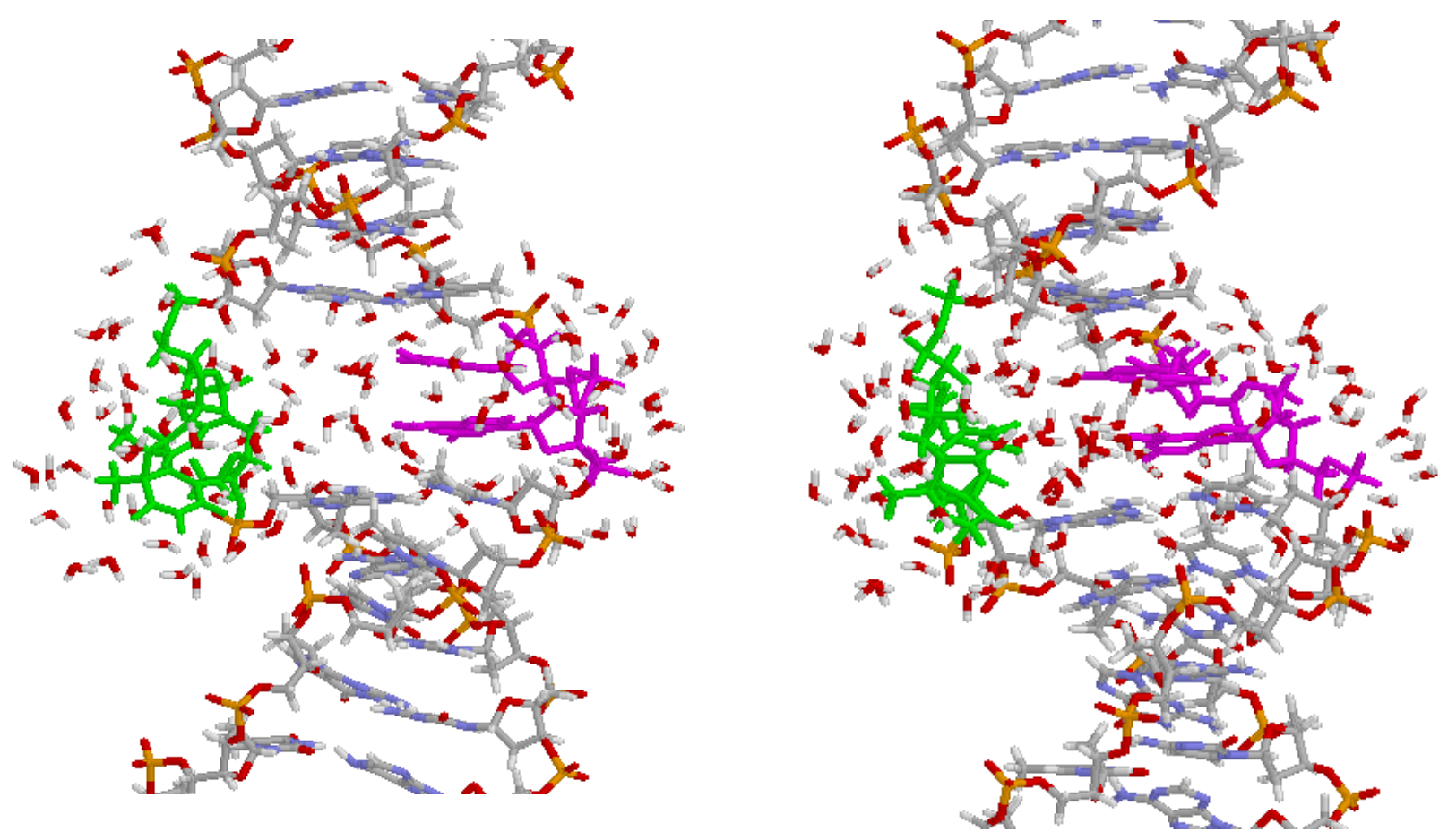

Figure S4. Flipped-out DNA structure snapshots of MD simulation at 3000 ps showing 50 closest waters (25 closest to orphaned adenines and 25 closest to CPD). Waters are positioned to hydrogen bond to the orphaned adenines in the "hole" left by the flipped-out CPD. Adenines are shown in pink, CPD in green. 
Table S1. Interactions between orphaned adenine bases and waters as calculated using ptraj. Data was calculated using 8000 frames of trajectory gathered over $8 \mathrm{~ns}$ of simulation. The interactions were calculated both over the entire $8 \mathrm{~ns}$ and also for $2 \mathrm{~ns}$ periods of time.

\begin{tabular}{|c|c|c|c|}
\hline Atom & Time period (ns) & \% occupancy & Distance $(\AA)$ \\
\hline Adenine $24, \mathrm{~N} 1$ & $0-8$ & 41.7 & $2.93 \pm 0.19$ \\
\hline Adenine $24, \mathrm{~N} 1$ & $0-2$ & 53.5 & $2.92 \pm 0.18$ \\
\hline Adenine $24, \mathrm{~N} 1$ & $2-4$ & 41.9 & $2.94 \pm 0.20$ \\
\hline Adenine $24, \mathrm{~N} 1$ & $4-6$ & 47.3 & $2.93 \pm 0.19$ \\
\hline Adenine $24, \mathrm{~N} 1$ & $6-8$ & 24.2 & $2.91 \pm 0.17$ \\
\hline Adenine $24, \mathrm{~N} 1$ & $0-8$ & 122.7 & $4.04 \pm 0.85$ \\
\hline Adenine $24, \mathrm{~N} 1$ & $0-2$ & 133.8 & $4.08 \pm 0.82$ \\
\hline Adenine $24, \mathrm{~N} 1$ & $2-4$ & 136.6 & $4.03 \pm 0.85$ \\
\hline Adenine $24, \mathrm{~N} 1$ & $4-6$ & 90.7 & $4.16 \pm 0.81$ \\
\hline Adenine $24, \mathrm{~N} 1$ & $6-8$ & 90.7 & $4.16 \pm 0.81$ \\
\hline Adenine 24, N6 & $0-8$ & 11.5 & $3.24 \pm 0.19$ \\
\hline Adenine $24, \mathrm{~N} 6$ & $0-2$ & 13.6 & $3.26 \pm 0.17$ \\
\hline Adenine $24, \mathrm{~N} 6$ & $2-4$ & 11.8 & $3.22 \pm 0.20$ \\
\hline Adenine $24, \mathrm{~N} 6$ & $4-6$ & 11.5 & $3.26 \pm 0.18$ \\
\hline Adenine $24, \mathrm{~N} 6$ & $6-8$ & 9.5 & $3.22 \pm 0.19$ \\
\hline Adenine 24, N6 & $0-8$ & 109.5 & $4.18 \pm 0.52$ \\
\hline Adenine $24, \mathrm{~N} 6$ & $0-2$ & 111.8 & $4.15 \pm 0.52$ \\
\hline Adenine $24, \mathrm{~N} 6$ & $2-4$ & 119.0 & $4.22 \pm 0.51$ \\
\hline Adenine $24, \mathrm{~N} 6$ & $4-6$ & 123.8 & $4.23 \pm 0.49$ \\
\hline Adenine $24, \mathrm{~N} 6$ & $6-8$ & 83.3 & $4.20 \pm 0.51$ \\
\hline Adenine $25, \mathrm{~N} 1$ & $0-8$ & 49.7 & $2.93 \pm 0.19$ \\
\hline Adenine $25, \mathrm{~N} 1$ & $0-2$ & 44.6 & $2.94 \pm 0.19$ \\
\hline Adenine $25, \mathrm{~N} 1$ & $2-4$ & 52.0 & $2.943 \pm 0.19$ \\
\hline Adenine $25, \mathrm{~N} 1$ & $4-6$ & 51.5 & $2.94 \pm 0.20$ \\
\hline Adenine $25, \mathrm{~N} 1$ & $6-8$ & 50.7 & $2.91 \pm 0.19$ \\
\hline Adenine $25, \mathrm{~N} 1$ & $0-8$ & 140.5 & $4.02 \pm 0.85$ \\
\hline Adenine $25, \mathrm{~N} 1$ & $0-2$ & 118.6 & $3.99 \pm 0.86$ \\
\hline Adenine $25, \mathrm{~N} 1$ & $2-4$ & 146.2 & $4.01 \pm 0.85$ \\
\hline Adenine $25, \mathrm{~N} 1$ & $4-6$ & 161.3 & $4.07 \pm 0.83$ \\
\hline Adenine $25, \mathrm{~N} 1$ & $6-8$ & 135.9 & $4.00 \pm 0.88$ \\
\hline Adenine $25, \mathrm{~N} 6$ & $0-8$ & 9.9 & $3.28 \pm 0.16$ \\
\hline Adenine $25, \mathrm{~N} 6$ & $0-2$ & 8.5 & $3.32 \pm 0.15$ \\
\hline Adenine $25, \mathrm{~N} 6$ & $2-4$ & 10.0 & $3.28 \pm 0.15$ \\
\hline Adenine $25, \mathrm{~N} 6$ & $4-6$ & 10.7 & $3.26 \pm 0.18$ \\
\hline Adenine $25, \mathrm{~N} 6$ & $6-8$ & 10.2 & $3.26 \pm 0.16$ \\
\hline Adenine $25, \mathrm{~N} 6$ & $0-8$ & 96.5 & $4.21 \pm 0.51$ \\
\hline Adenine $25, \mathrm{~N} 6$ & $0-2$ & 84.3 & $4.21 \pm 0.50$ \\
\hline Adenine $25, \mathrm{~N} 6$ & $2-4$ & 113.2 & $4.23 \pm 0.50$ \\
\hline Adenine $25, \mathrm{~N} 6$ & $4-6$ & 105.6 & $4.21 \pm 0.52$ \\
\hline Adenine $25, \mathrm{~N} 6$ & $6-8$ & 82.9 & $4.18 \pm 0.52$ \\
\hline
\end{tabular}


Curves Analysis

For both simulations, flipped-in and flipped-out, a pdb file was output every 80 ps and used as the input for Curves. The global bend vs. time is shown below for both simulations. A similar procedure was followed for the corresponding non-damaged structure ( 2 ns MD production runs, global bend calculated at $1200 \mathrm{ps}$ ) and the global bend was $7.2^{\circ}$.

Figure S5. Flipped-in structure.

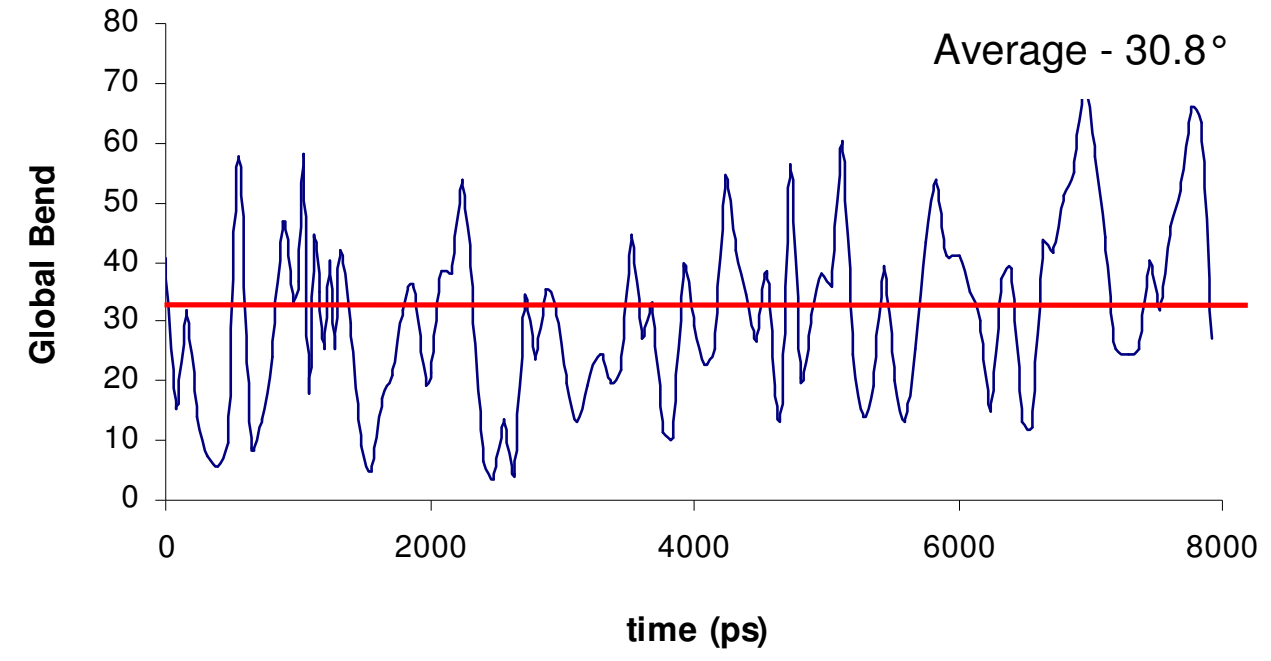

Figure S6. Flipped-out structure.

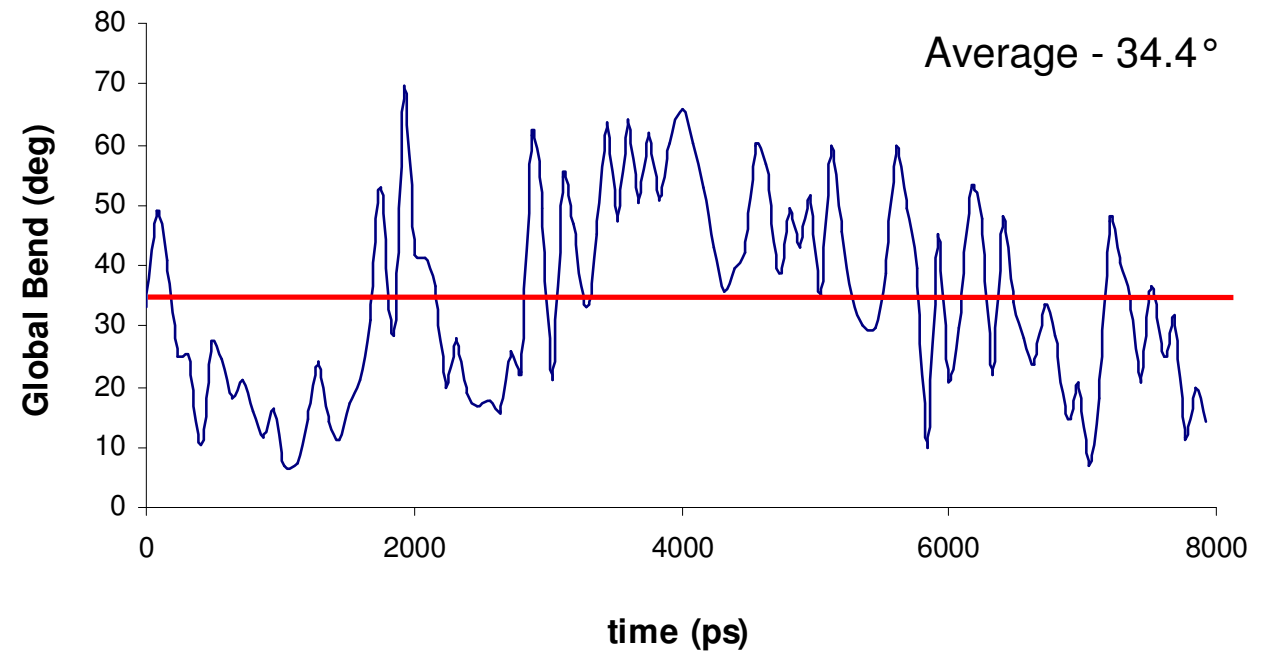


Snapshots of flipped-out DNA structures corresponding to 5' pseudodihedral angles of $139^{\circ}$ and $170^{\circ}$. In both structures the thymine dimer is flipped-out of the duplex but for the less common structure ( $5^{\prime}$ pseudodihedral $170^{\circ}$ ) the dimer is facing the major groove.
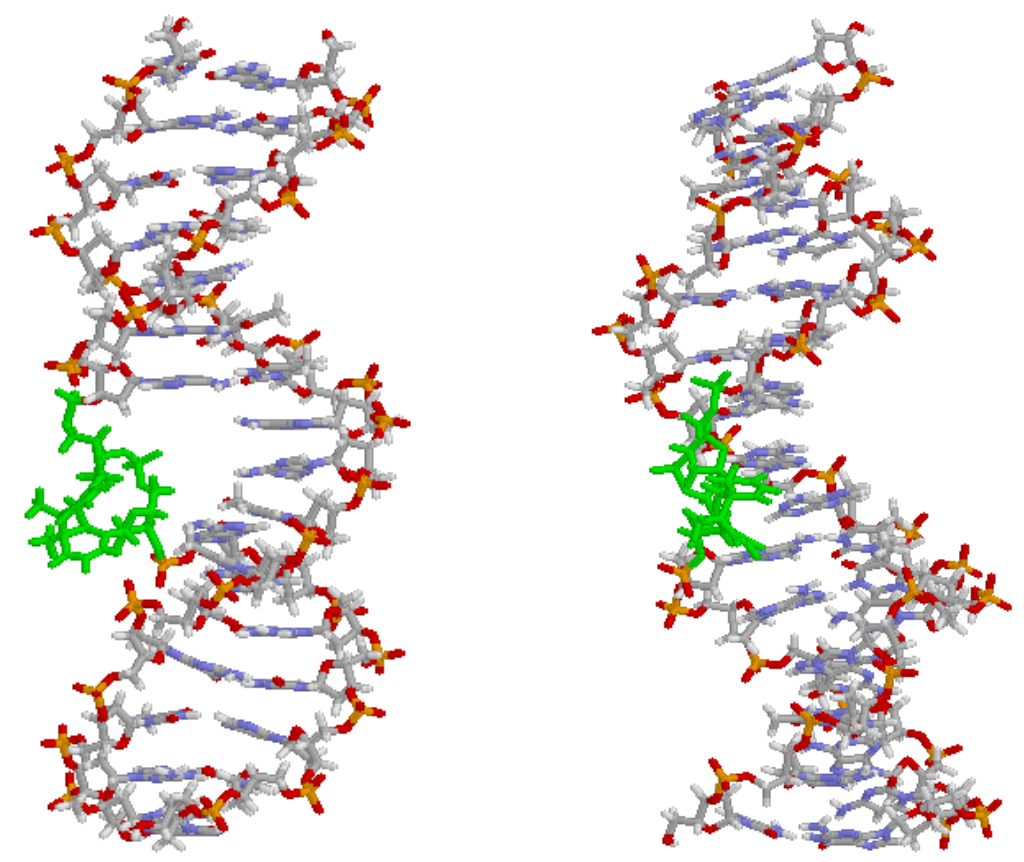

Figure S7. Flipped-out DNA structure snapshot of MD simulation at 3000 ps. 5' pseudodihedral angle $=139^{\circ}$.
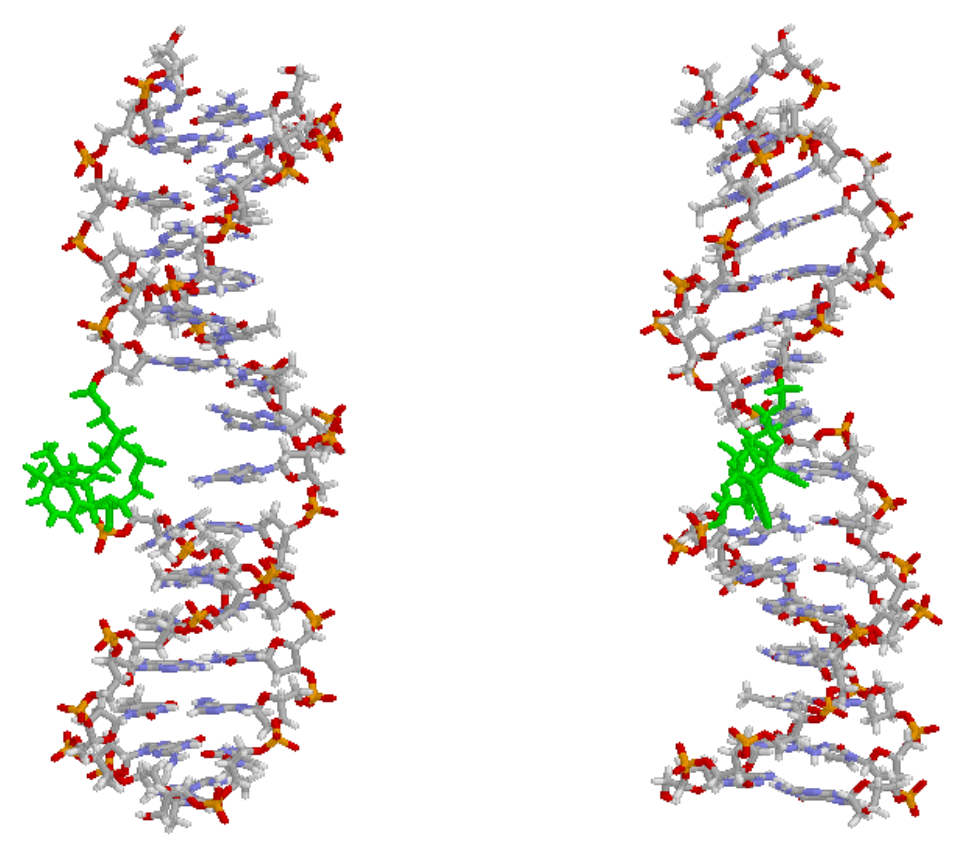

Figure S8. Flipped-out DNA structure snapshot of MD simulation at 3500 ps. 5' pseudodihedral angle $=170^{\circ}$. 
Detailed view of sampling scheme used to generate base flipping PMF. Top, same as shown in Figure 9 in Methods section of text. Large squares, 3 ns equilibration, 2.5 ns sampling. Other points, $100 \mathrm{ps}$ sampling, $2500 \mathrm{~ns}$ sampling. Open squares, base flipping coordinate. Filled circles, sampling extensions to move artificially high energy values away from the desired coordinate.

Figure S9. Large points, 3 ns sampling points. Surrounding points were started from the extensively sampled points to remove the risk of hysteresis.

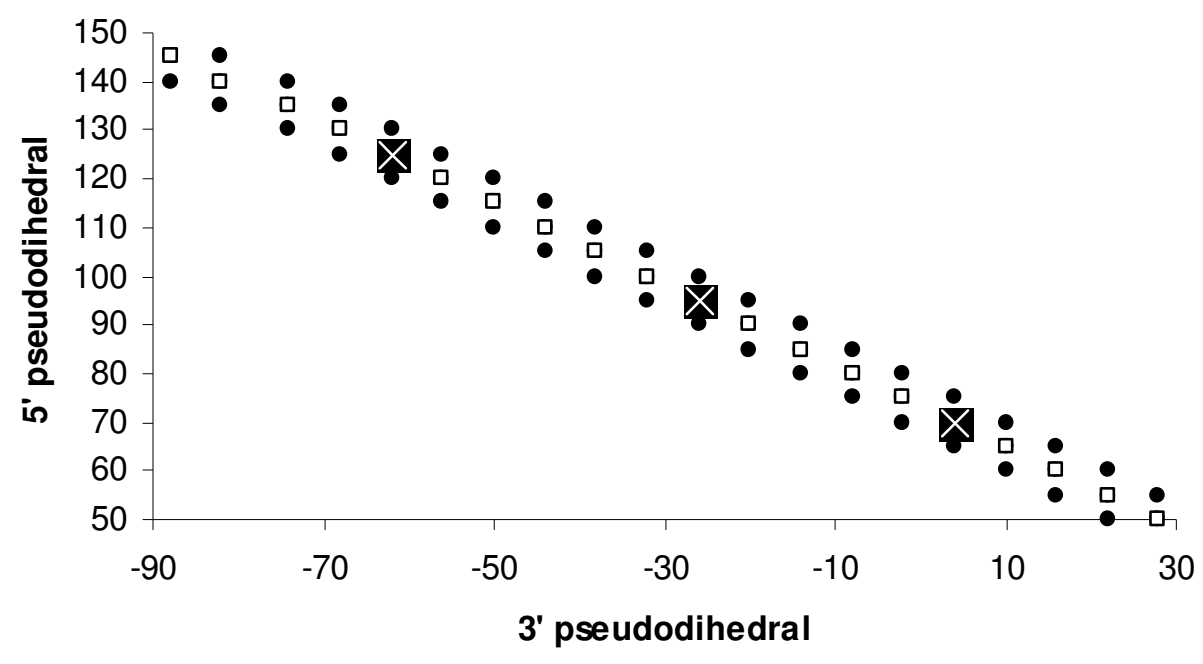

Figure S10. Sampling scheme showing the starting points for the simulation windows. The outlined point are the extensively equilibrated, $3 \mathrm{~ns}$, structures. The adjacent points of similar color were started from the corresponding extensively equilibrated structure

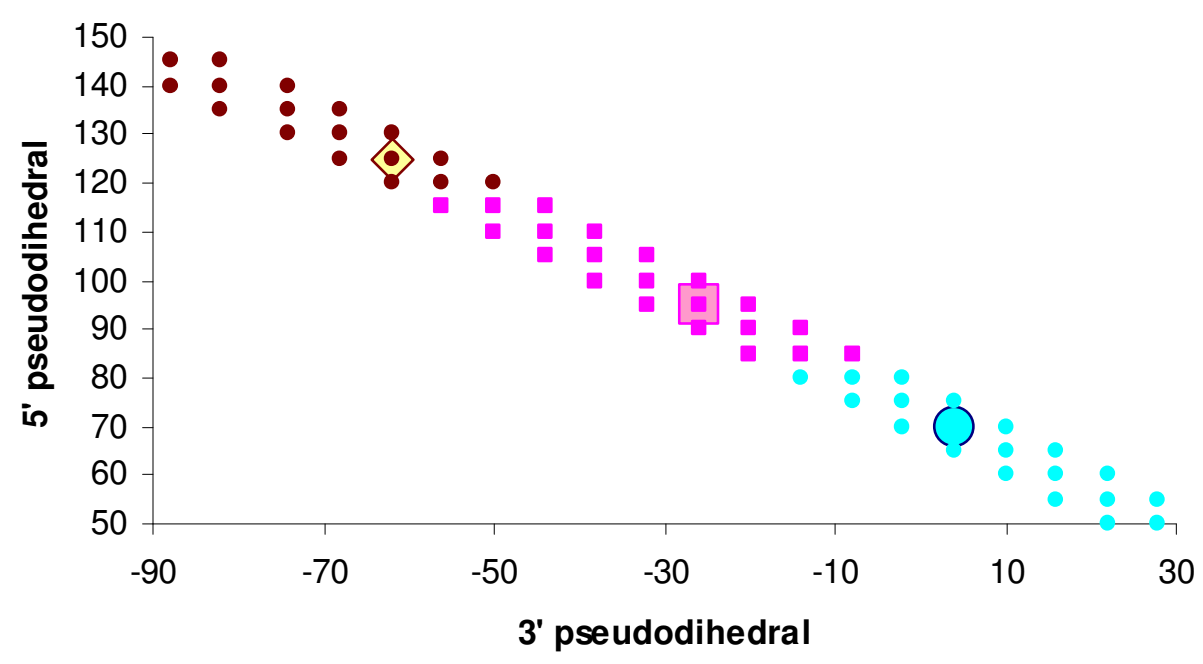

\section{Convergence of PMF}


In order to determine the convergence the difference between the PMFs calculated with the two equilibration and sampling times was computed as is shown in a surface representation. In all graphs there are high energy spikes caused by the absence of sampling in one bin for one equilibration, sampling time and the presence of sampling in the other. Convergence was achieved as indicated by the small difference in free energies of the two equilibration, sampling time sets.

Figure S11. Calculated using a $2.5^{\circ}$ bin width for the $5^{\prime}$ pseudodihedral and a $3^{\circ}$ bin width for the $3^{\prime}$ pseudodihedral. This is necessary due to the shorter sampling time.

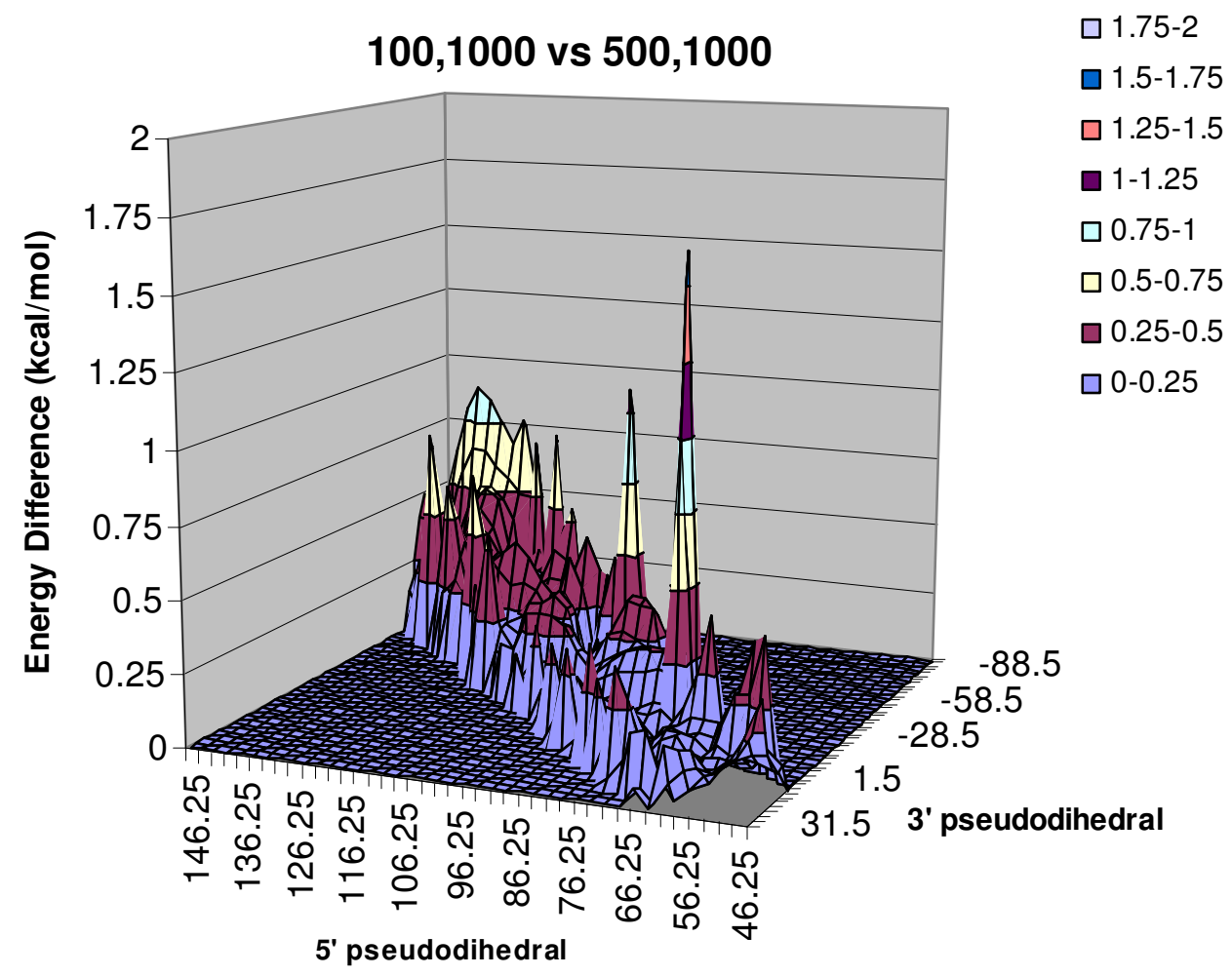


Figure S12. Calculated using a $2.5^{\circ}$ bin width for the $5^{\prime}$ pseudodihedral and a $3^{\circ}$ bin width for the $3^{\prime}$ pseudodihedral. This is necessary due to the shorter sampling time. While the energy difference between the 100 ps and 500 ps equilibration times is larger than for the 100 ps and 200 ps equilibration times it is still acceptable, especially given the reduction in computation time. Therefore, the equilibration time used for the computation of the final PMF was 100 ps.

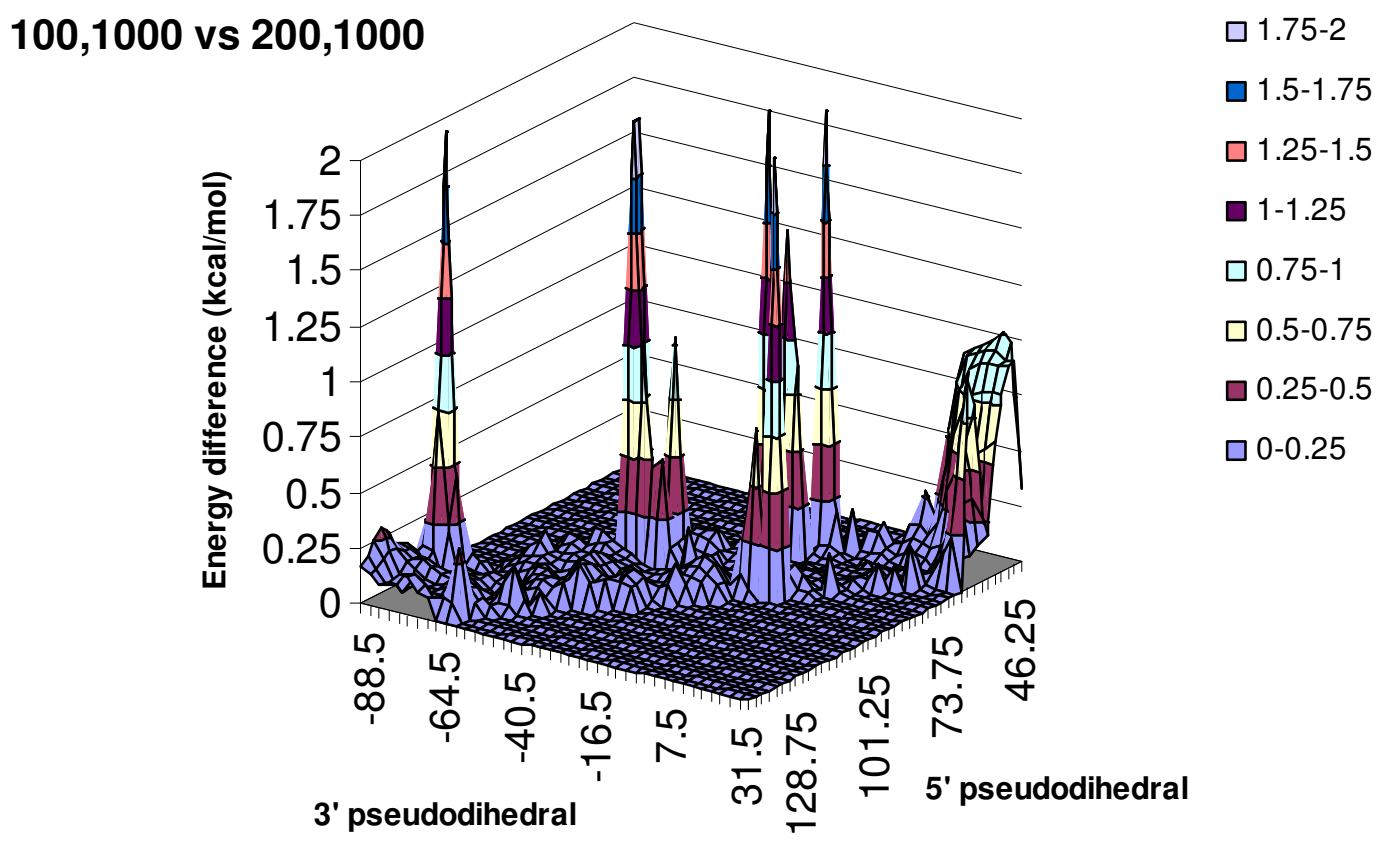

Figure S13. Calculated using a $2.5^{\circ}$ bin width for the $5^{\prime}$ pseudodihedral and a $3^{\circ}$ bin width for the $3^{\prime}$ pseudodihedral. This is necessary due to the shorter sampling time. 
Supplementary Information for: O’Neil, L.L., Grossfield, A. \& Wiest, O.: On the Base Flipping... S10

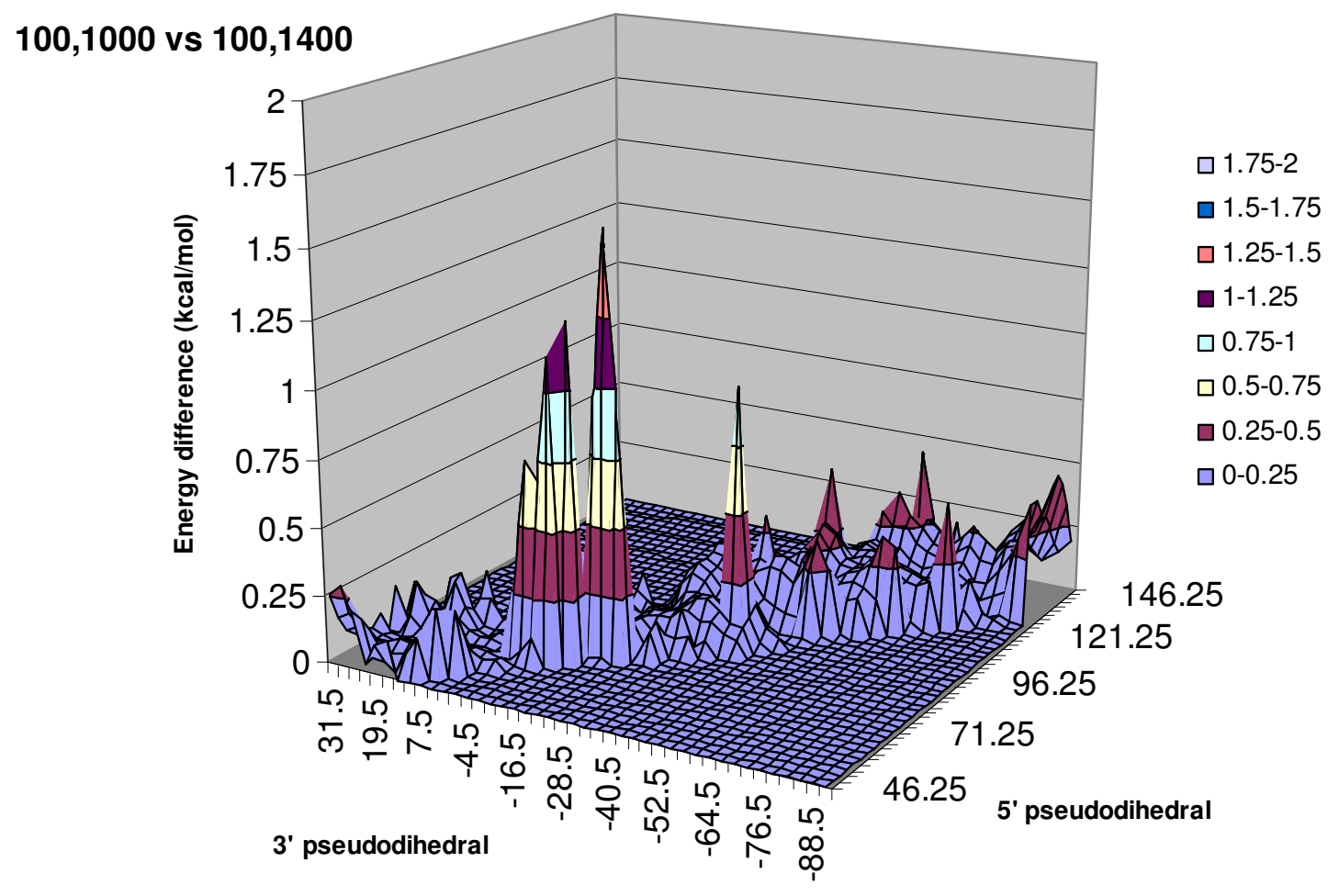

Figure S14. Computed using $0.5^{\circ}$ bin widths for the 5' pseudodihedral and $0.6^{\circ}$ bin widths for the $3^{\prime}$ pseudodihedral. Using larger bin widths for the data generated using $2.5 \mathrm{~ns}$ sampling results in loss of resolution of data. The majority of the sampled region has an energy difference of $0-0.1$ $\mathrm{kcal} / \mathrm{mol}$ or $0.1-0.2 \mathrm{kcal} / \mathrm{mol}$ indicating convergence. 
Supplementary Information for: O’Neil, L.L., Grossfield, A. \& Wiest, O.: On the Base Flipping... S11

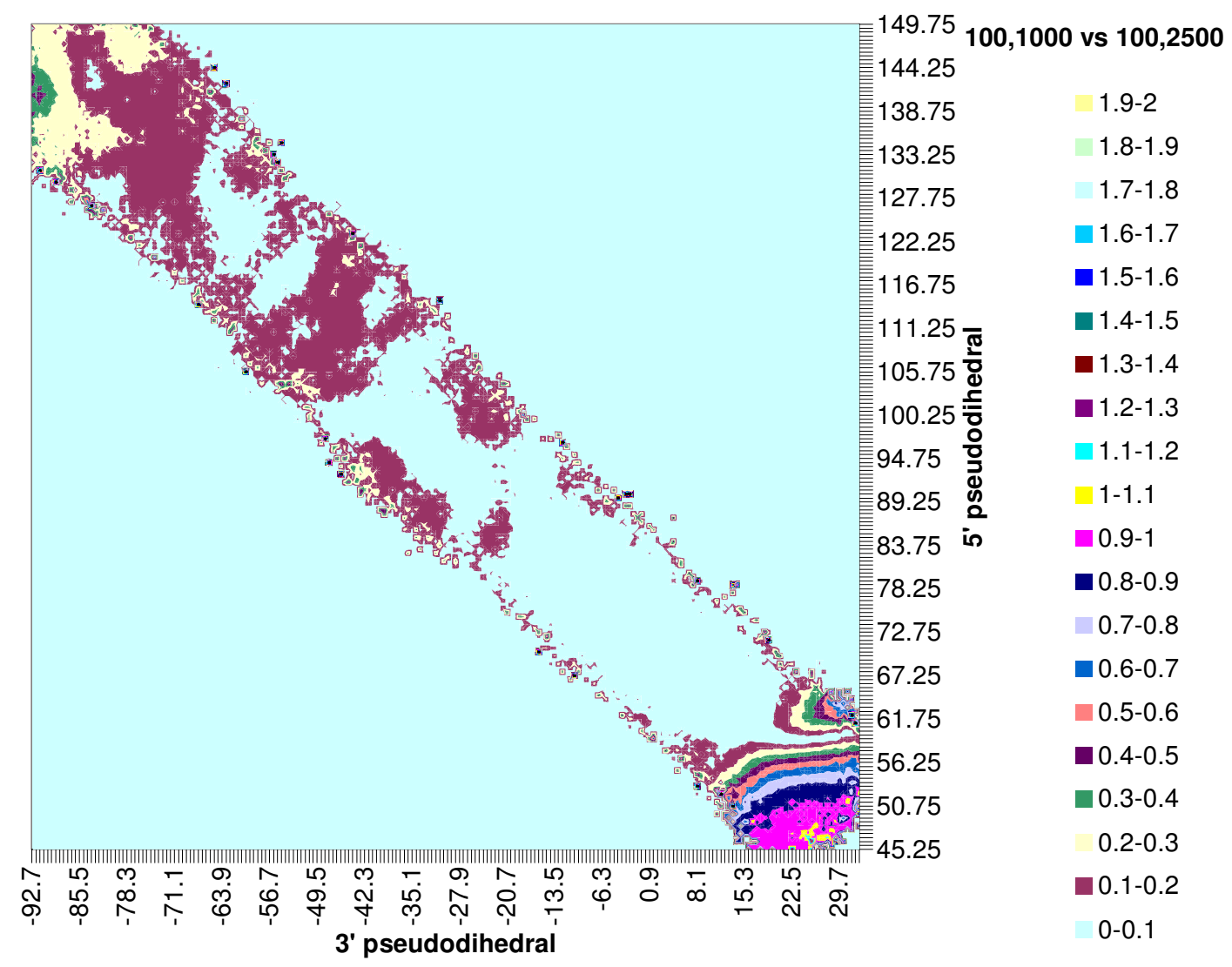

